\title{
Highly efficient tandem $p-i-n$ organic light-emitting diodes adopting a low temperature evaporated rhenium oxide interconnecting layer
}

\author{
Dong-Seok Leem, ${ }^{1}$ Jae-Hyun Lee, ${ }^{1}$ Jang-Joo Kim, ${ }^{1, a)}$ and Jae-Wook Kang ${ }^{2}$ \\ ${ }^{1}$ Department of Materials Science and Engineering and Center for Organic Light Emitting Diode, \\ Seoul National University, Seoul 151-744, Republic of Korea \\ ${ }^{2}$ Department of Surface Technology, Korea Institute of Materials and Science (KIMS), Changwon 641-831 \\ Republic of Korea
}

(Received 13 June 2008; accepted 19 August 2008; published online 8 September 2008)

\begin{abstract}
High quality interconnection units (ICUs) with a high transparency and superior charge generating capability for tandem organic light-emitting diodes (OLEDs) are developed. The ICUs of rubidium carbonate-doped 4,7-diphenyl-1,10-phenanthroline/rhenium oxide $\left(\mathrm{ReO}_{3}\right)$-doped $N, N^{\prime}$-diphenyl- $N, N^{\prime}$-bis(1, 1'-biphenyl)-4, $4^{\prime}$-diamine layers with or without an additional $\mathrm{ReO}_{3}$ interlayer produce high transmittance $(88 \%-92 \%$ at $420-700 \mathrm{~nm})$ and spontaneous internal charge generation properties. A very high efficiency of $\sim 129 \mathrm{~cd} / \mathrm{A}$ has been demonstrated from only two stacked green $p-i$ - $n$ OLEDs by employing the developed ICUs. The relationship between the device efficiency and internal charge generation within the ICUs is further described by means of the capacitance measurements. (C) 2008 American Institute of Physics. [DOI: 10.1063/1.2979706]
\end{abstract}

Stacked (tandem) organic light-emitting diodes (OLEDs) are attractive for next-generation displays and solid-state lightings owing to their significantly high current efficiency and brightness. ${ }^{1-15}$ High performance tandem OLEDs require formation of high quality interconnection unit (ICU) possessing high optical transparency as well as superior charge generation and injection properties. ${ }^{1-5}$ Researchers have reported on several types of ICUs including metalbased multilayers, ${ }^{2,6}$ undoped organic bilayers, ${ }^{3}$ organicdoped organic $p-n$ junctions, $^{7-9}$ and doped organic/metal oxide bilayers. ${ }^{4,10-14}$ Among them, the use of metal-based ${ }^{2,6}$ or undoped organic bilayer ICUs (Ref. 3) are feasible for simple fabrication of tandem OLEDs. However, the relatively low transmittance (below $70 \%$ in the visible range) of metal-based ICUs (Refs. 2 and 6) and high operation voltages of tandem devices employing undoped organic bilayer ICUs (Ref. 3) remain as problems. On the other hand, metal and organic doped $n-p$ junction ICUs, ${ }^{7-9}$ such as Bphen: $\mathrm{Cs} / N, N^{\prime}-$ Di(naphthalen - 1 - yl)- $N, N^{\prime}$ - diphenyl- benzidine : tetrafluorotetracyano-quinodimethane $\left(\mathrm{F}_{4}-\mathrm{TCNQ}\right){ }^{8}$ and $\mathrm{Alq}_{3}: \mathrm{Mg} / \mathrm{m}$ MTDATA: $\mathrm{F}_{4}-\mathrm{TCNQ}{ }^{9}$ have been widely used to fabricate efficient tandem OLEDs due to their good electrical and optical properties. However, recent results have shown that the highly diffusive $p$-dopant ( $\mathrm{F}_{4}$-TCNQ) (Refs. 3 and 15$)$ decreases interfacial stability causing an increase in drive voltage during operation. ${ }^{15}$ Unlike the organic doping method, the application of relatively stable metal oxides as connection systems such as $\mathrm{Alq}_{3}: \mathrm{Mg} / \mathrm{WO}_{3},{ }^{10} \mathrm{Alq}_{3}: \mathrm{Mg} / \mathrm{V}_{2} \mathrm{O}_{5},{ }^{11}$ $\mathrm{Alq}_{3}: \mathrm{Cs}_{2} \mathrm{CO}_{3} / \mathrm{MoO}_{3}{ }^{12}$ and Bphen: $\mathrm{Cs}_{2} \mathrm{CO}_{3} / \mathrm{NPB}: \mathrm{WO}_{3}$, ${ }^{13}$ also have been developed. The metal oxides, themselves, can produce good electrical conductivity and a high transmittance of $85 \%-90 \%$ in the visible range, ${ }^{4}$ but their high evaporation temperatures (over $600{ }^{\circ} \mathrm{C}$ ) diminish compatibility with organic molecules and hinder their practical application in tandem devices. $2,3,9,15$

\footnotetext{
a) Author to whom correspondence should be addressed. TEL.: +82-2-8807893. FAX: +82-2-889-8702. Electronic mail: jjkim@snu.ac.kr.
}

In this letter, we report on highly transparent and superior charge generating organic $p-n$ junction ICUs. The developed ICUs consist of rubidium carbonate $\left(\mathrm{Rb}_{2} \mathrm{CO}_{3}\right)$-doped 4,7-diphenyl-1,10-phenanthroline (Bphen)/rhenium oxide $\left(\mathrm{ReO}_{3}\right)$-doped $\quad N, N^{\prime}$-diphenyl- $N, N^{\prime}$-bis(1, $1^{\prime}$-biphenyl)$4,4^{\prime}$-diamine (NPB) with and without a very thin $\mathrm{ReO}_{3}$ interlayer. The developed ICUs showed a high transmittance of $88 \%-92 \%$ at $420-700 \mathrm{~nm}$ wavelengths. Excess absorption by the doping and the $\mathrm{ReO}_{3}$ interlayer was below $4 \%$ in the entire visible range. A high efficiency of $129 \mathrm{~cd} / \mathrm{A}$ was obtained from two stacked phosphorescent green $p-i-n$ OLEDs by employing a developed ICU, which was mainly attributed to the superior internal charge generation as well as the high transparency of the ICU.

The patterned indium tin oxide (ITO) substrates were prepared and basically cleaned. ${ }^{16}$ Before construction of the two stacked $p-i$ - $n$ OLEDs, the single $p-i$ - $n$ OLED structure was fabricated and optimized. This structure consisted of the ITO anode, 4 wt $\% \mathrm{ReO}_{3}$-doped NPB hole transporting layer (HTL) $(80 \mathrm{~nm})$, undoped NPB $(20 \mathrm{~nm})$, a double emission layer $(30 \mathrm{~nm})$ of $8 \mathrm{wt} \% \quad \operatorname{Ir}(\text { ppy })_{3}$-doped 4-4' $-N, N^{\prime}$-dicarbazolylbiphenyl and $8 \mathrm{wt} \% \operatorname{Ir}(\mathrm{ppy})_{3}$-doped Bphen, undoped Bphen (40 nm), 15 wt \% $\mathrm{Rb}_{2} \mathrm{CO}_{3}$-doped Bphen electron transporting layer $(15 \mathrm{~nm})$, and an Al cathode. After that, we fabricated tandem $p-i-n$ OLEDs by simply stacking the optimized single $p-i-n$ cell with or without a $\mathrm{ReO}_{3}$ interlayer between the $n-p$ junction ICUs. The structures of the devices are schematically shown in Fig. 1. We employed $\mathrm{ReO}_{3}$ as the $p$-dopant and the interlayer because it can be evaporated at a lower temperature $\left(\sim 340{ }^{\circ} \mathrm{C}\right)$ than $\mathrm{MoO}_{3}\left(\sim 620{ }^{\circ} \mathrm{C}\right)$, alleviating the drawbacks of metal oxides in a practical manufacturing process induced by a high evaporation temperature. ${ }^{15}$ Moreover, the device stability can be enhanced by doping $\mathrm{ReO}_{3}$ in HTL; and the doping capability of $\mathrm{ReO}_{3}$ is superior to other metal oxide dopants. ${ }^{16}$ The current density-voltage-luminance $(J-V-L)$ characteristics of the devices were measured by a Keithley 2400 semiconductor parameter analyzer and a Photo Research (PR-650) spectrophotometer. We measured the angular-dependent electroluminescence (EL) spectra by an optical fiber and a S2000 


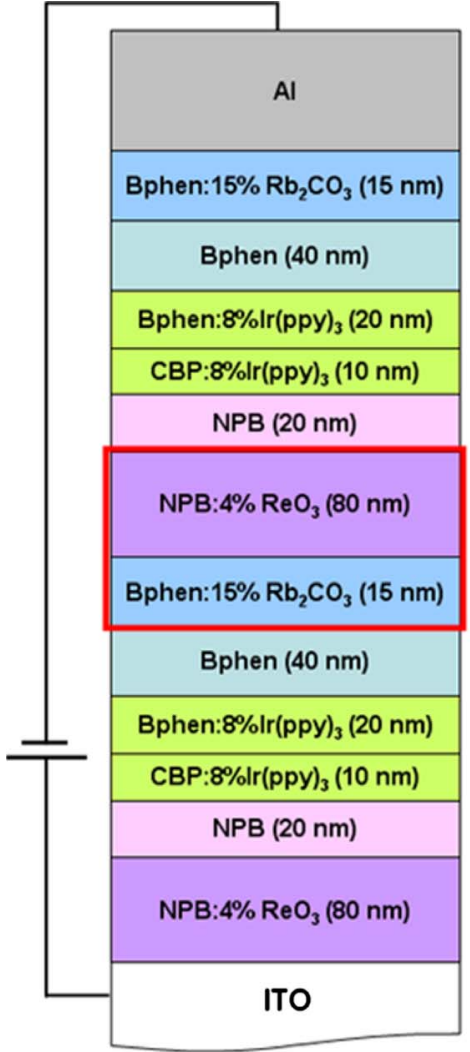

Tandem A

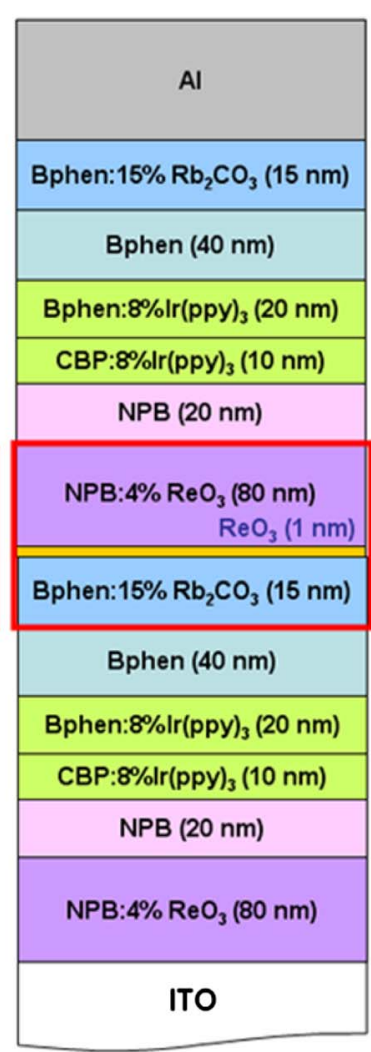

Tandem B
FIG. 1. (Color online) The schematic illustration of tandem $p-i$ - $n$ OLEDs

miniature fiber optic spectrometer (Ocean Optics). The capacitance-voltage measurements of the ICUs were carried out by a 1260 impedance/gain-phase analyzer and a 1287 electrochemical interface (Solartron). The transmittance was measured by means of a UV-visible spectrophotometer (Cary 5000).

Figure 2(a) shows the $J$ - $V$ - $L$ characteristics of single and tandem OLEDs. The two stacked OLEDs with the doped organic $p$ - $n$ junction ICU (tandem A) exhibits an operation voltage of $9.5 \mathrm{~V}$ at $1000 \mathrm{~cd} / \mathrm{m}^{2}$, which is larger than twice that of the operation voltage $(3.6 \mathrm{~V})$ of a single OLED. However, the incorporation of a very thin $(1 \mathrm{~nm}) \mathrm{ReO}_{3}$ interlayer at the interface between the $n$-doped and $p$-doped layer in the ICU (tandem B) significantly reduced the operation voltage to $8.1 \mathrm{~V}$ at $1000 \mathrm{~cd} / \mathrm{m}^{2}$, indicating that the insertion of a thin $\mathrm{ReO}_{3}$ layer effectively enhances the electrical properties of the doped organic $p-n$ junction ICU. In addition to that, the tandem $\mathrm{B}$ device shows a very high luminance of $68000 \mathrm{~cd} / \mathrm{m}^{2}$ at a current density of $242 \mathrm{~mA} / \mathrm{cm}^{2}$ and a bias voltage of $14.8 \mathrm{~V}$ (not shown). The current efficiency of single and tandem OLEDs are displayed in Fig. 2(b) as a function of the current density. A very high current efficiency of $129 \mathrm{~cd} / \mathrm{A}$ is obtained from the tandem $\mathrm{B}$, which is almost twice that of the single OLED ( $68 \mathrm{~cd} / \mathrm{A})$. The tandem A device also exhibits a high current efficiency of $111 \mathrm{~cd} / \mathrm{A}$, but a little lower than that of the tandem B.

Figure 3 displays the EL spectra of single and tandem $p-i-n$ OLEDs. The stacked OLEDs (A and B) exhibit almost the same EL spectra as a single device without any spectrum narrowing. The EL spectra of tandem OLEDs also show no angle dependence and the emission intensity follows Lam-
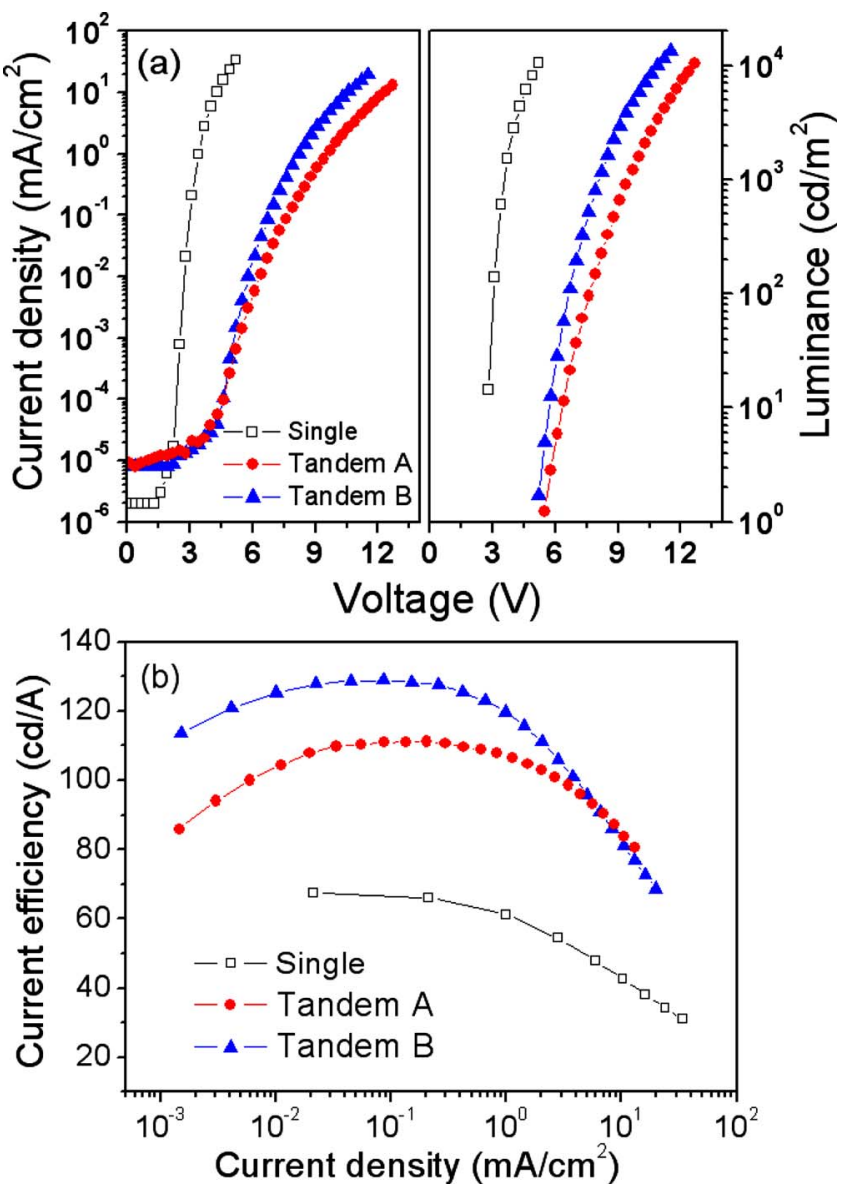

FIG. 2. (Color online) (a) The current density-voltage-luminance characteristics of single and tandem $p-i-n$ OLEDs. (b) The current efficiency-current density plots of single and tandem OLEDs.

bertian distribution (the inset of Fig. 3). These results suggest that the two types of ICUs are optically transparent, and minimize the microcavity effect. The microcavity effect results in EL spectrum narrowing, angle dependent spectral change, and deviation from the Lambertian distribution of emission intensity. This microcavity effect was commonly observed in tandem devices employing metal-based ICUs where the microcavity is formed by metal-based semireflective ICUs and a highly reflective metal electrode (cathode). ${ }^{6}$ In contrast, our devices show little microcavity effect due to the high transmittance (low reflectivity) of the ICUs.

We proved high optical transparency of the ICUs by a transmittance measurement, as shown in Fig. 4. Both ICUs

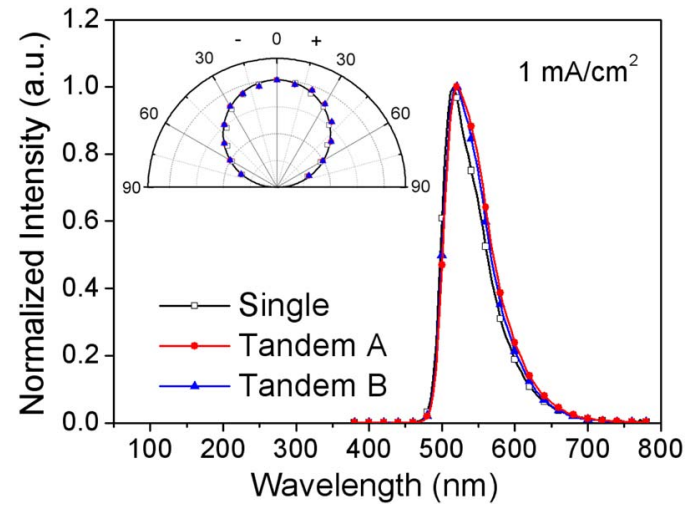

FIG. 3. (Color online) The EL spectra of single and tandem $p-i-n$ OLEDs. The inset depicts the normalized emission patterns of devices. (Line indicates Lambertian distribution). 


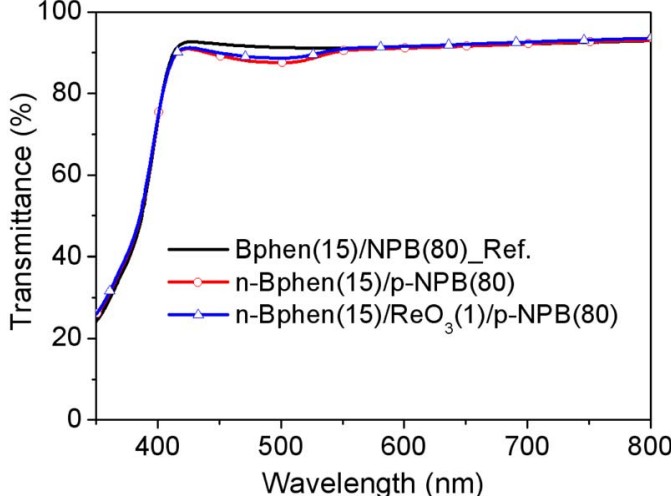

FIG. 4. (Color online) The transmittance of Bphen: $\mathrm{Rb}_{2} \mathrm{CO}_{3}(15 \mathrm{~nm}) / \mathrm{NPB}: \mathrm{ReO}_{3} \quad(80 \mathrm{~nm}) \quad$ (open circle), Bphen: $\mathrm{Rb}_{2} \mathrm{CO}_{3}(15 \mathrm{~nm}) / \mathrm{ReO}_{3}(1 \mathrm{~nm}) / \mathrm{NPB}: \mathrm{ReO}_{3}(80 \mathrm{~nm})$ (open triangle) ICUs, and the reference of Bphen $(15 \mathrm{~nm}) / \mathrm{NPB}(80 \mathrm{~nm})$ (line).

with and without a thin $\mathrm{ReO}_{3}$ interlayer exhibit a high transmittance of $88 \%-92 \%$ at wavelengths of $420-700 \mathrm{~nm}$, which is much higher than that (about 60\%) of common metallic ICUs. ${ }^{2}$ The reference undoped Bphen/NPB bilayers also show a high transmittance of $91 \%-92 \%$ at the same wavelengths. The excess loss of transmittance by doping and an interlayer is less than $4 \%$, which is mainly originated from the increase in absorption by the formation of charge transfer complexes between the NPB and $\mathrm{ReO}_{3} \cdot{ }^{16}$ Little change in the absorption is induced by the doping of $\mathrm{Rb}_{2} \mathrm{CO}_{3}$ in Bphen. This transparency is much higher than that of an ICU using $\mathrm{MoO}_{3}{ }^{4}$

Since the optical transparency of the two ICUs are almost the same, the enhancement in efficiency by the incorporation of a very thin $\mathrm{ReO}_{3}$ interlayer must be associated with effective generation of additional charge carriers within the ICUs and subsequent carrier injection and transport from the ICUs into the stacked $p-i-n$ cells. We investigated the effectiveness and possible internal charge generation of the ICUs by means of capacitance measurements. ${ }^{17,18}$

Figure 5 shows capacitance-voltage $(C-V)$ characteristics of the developed ICUs. Details about the ICU structures are schematically illustrated in the inset of Fig. 4. Note that a $50 \mathrm{~nm}$ thick insulating LiF layer was deposited above and below the ITO anode and $\mathrm{Al}$ cathode, respectively, in order to prevent external charge injection from the electrodes. The reference sample with an undoped organic bilayer exhibits

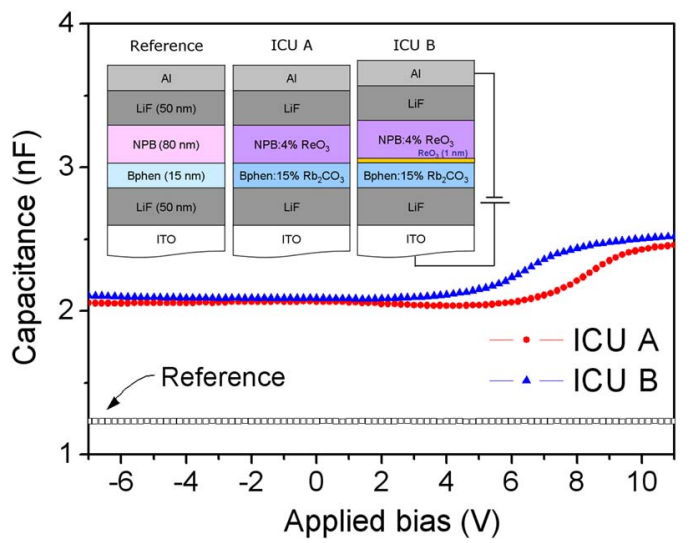

FIG. 5. (Color online) The capacitance-voltage characteristics of ICUs measured at the fixed frequency of $1000 \mathrm{~Hz}$. The inset displays the details of ICUs. no change in capacitance with the applied voltages, indicating no generation of internal charge carriers. The samples with ICUs A and B, however, show higher capacitance than that of the reference even at low and negative (forward for the $n-p$ junction) bias. We attribute this higher capacitance to the larger dielectric constants of the doped layers coming from the larger polarization of the charge transfer complexes. In addition to that, the capacitances apparently increase with the increment of positive (reverse for the $n-p$ junction) bias above certain voltages for the devices. These results suggest that additional (internal) charges are generated within the ICUs since no charges can be injected into the ICUs due to the thick LiF insulating layers. ${ }^{17,18} \mathrm{We}$ note that the capacitance of ICU B begins to increase at a lower bias voltage and is higher under the same applied bias than that of ICU A. This fact suggests that the incorporation of a very thin $\mathrm{ReO}_{3}$ layer in the ICU B generates more charges at a lower voltage than the ICU A and consequently contribute to the enhancement in efficiency as well as the lowering in an operation voltage of tandem OLED B (Fig. 2). Our quantitative analysis of the charge generation efficiency in the ICUs will be published elsewhere.

In summary, we demonstrated high efficiency green tandem OLEDs using Bphen: $\mathrm{Rb}_{2} \mathrm{CO}_{3} / \mathrm{NPB}: \mathrm{ReO}_{3}$ and Bphen: $\mathrm{Rb}_{2} \mathrm{CO}_{3} / \mathrm{ReO}_{3} / \mathrm{NPB}: \mathrm{ReO}_{3}$ as ICUs. Two stacked OLEDs adopting one of the ICUs showed a very high efficiency of $\sim 129 \mathrm{~cd} / \mathrm{A}$ with little angular dependence of the EL spectrum, resulting from high optical transmittance $(88-92 \%$ at $420-700 \mathrm{~nm})$ and efficient charge generation of the ICUs. The insertion of a thin $\mathrm{ReO}_{3}$ interlayer within the ICUs was very effective in generating charges as demonstrated by the $C-V$ measurement and in lowering the operation voltage of the stacked OLEDs.

The authors thank the MKE of Korea and Samsung SDI for their financial support of this work.

${ }^{1}$ M.-Y. Chan, S.-L. Lai, K.-M. Lau, M.-K. Fung, C.-S. Lee, and S.-T. Lee, Adv. Funct. Mater. 17, 2509 (2007).

${ }^{2}$ H. Zhang, Y. Dai, and D. Ma, Appl. Phys. Lett. 91, 123504 (2007).

${ }^{3}$ S. L. Lai, M. Y. Chan, M. K. Fung, C.-S. Lee, and S. T. Lee, J. Appl. Phys. 101, 014509 (2007).

${ }^{4}$ H. Kanno, R. J. Holmes, Y. Sun, S. Kena-Cohen, and S. R. Forrest, Adv. Mater. (Weinheim, Ger.) 18, 339 (2006).

${ }^{5}$ H. Kanno, Y. Hamada, K. Nishimura, K. Okumoto, N. Saito, H. Ishida, H. Takahashi, K. Shibata, and K. Mameno, Jpn. J. Appl. Phys., Part 1 44, 9219 (2006)

${ }^{6}$ J. X. Sun, X. L. Zhu, H. J. Peng, M. Wong, and H. S. Kwok, Appl. Phys. Lett. 87, 093504 (2005).

${ }^{7}$ L. S. Liao, K. P. Klubek, and C. W. Tang, Appl. Phys. Lett. 84, 167 (2004)

${ }^{8}$ T.-Y. Cho, C.-L. Lin, and C.-C. Wu, Appl. Phys. Lett. 88, 111106 (2006).

${ }^{9}$ C. W. Law, K. M. Lau, M. K. Fung, M. Y. Chan, F. L. Wong, C. S. Lee, and S. T. Lee, Appl. Phys. Lett. 89, 133511 (2006).

${ }^{10}$ C.-C. Chang, S.-W. Hwang, C. H. Chen, and J.-F. Chen, Jpn. J. Appl. Phys., Part 1 43, 6418 (2004).

${ }^{11}$ T. Tsutsui and M. Terai, Appl. Phys. Lett. 84, 440 (2004).

${ }^{12}$ C.-W. Chen, Y.-J. Lu, C.-C. Wu, E. H.-E. Wu, C.-W. Chu, and Y. Yang, Appl. Phys. Lett. 87, 241121 (2005).

${ }^{13}$ M.-H. Ho, T.-M. Chen, P.-C. Yeh, S.-W. Hwang, and C. H. Chen, Appl. Phys. Lett. 91, 233507 (2007).

${ }^{14}$ F. Guo and D. Ma, Appl. Phys. Lett. 87, 173510 (2005).

${ }^{15}$ L.-S. Liao, W. K. Slusarek, T. K. Hatwar, M. L. Ricks, and D. L. Comfort, Adv. Mater. (Weinheim, Ger.) 20, 324 (2008).

${ }^{16}$ D.-S. Leem, H.-D. Park, J.-W. Kang, J.-H. Lee, J. W. Kim, and J.-J. Kim, Appl. Phys. Lett. 91, 011113 (2007).

${ }^{17}$ M. Terai and T. Tsutsui, Appl. Phys. Lett. 90, 083502 (2007).

${ }^{18}$ M. Kroger, S. Hamwi, J. Meyer, T. Dobbertin, T. Riedl, W. Kowalsky, and H.-H. Jonannes, Phys. Rev. B 75, 235321 (2007). 